\title{
Conséquences qualitatives de la sécheresse sur l'alimentation en eau potable
}

\author{
Mme M. Rizet \\ Laboratoire central de la Lyonnaise des eaux, Le Pecq
}

Sous nos climats tempérés froids, l'abondance moyenne des précipitations, environ 500 à $800 \mathrm{~mm} / \mathrm{an}$, compense largement les pertes en eau par évaporation ou évapotranspiration.

Toutefois, cette moyenne est sujette à d'importantes fluctuations dans le temps (tabl. l) et dans l'espace.

Tableau 1. Moyenne mensuelle des précipitations en région parisienne ouest

\begin{tabular}{|c|c|c|}
\hline \multirow{2}{*}{ MoIs } & \multicolumn{2}{|c|}{ PRECIPITATIONS EN MM } \\
\hline & 1976 & 1965 \\
\hline & & \\
01 & 17,7 & 95,8 \\
2 & 32,6 & 5,5 \\
3 & 15,7 & 73,8 \\
4 & 12,5 & 39,3 \\
5 & 1,4 & 78,1 \\
6 & 64,3 & 67,1 \\
7 & 59,3 & 73,7 \\
8 & 29,3 & 120,6 \\
9 & 36,2 & 19,4 \\
10 & & 105,6 \\
11 & & 99,6 \\
\hline
\end{tabular}

Des réservoirs en barrage ou en dérivation ont été créés pour tamponner ces variations (écrêtement des crues, compensation des étiages). Nous verrons toutefois dans ce dernier cas qu'un tel remède hydraulique peut être une source de perturbation qualitative.

Une période de sécheresse va interférer à plusieurs titres sur la qualité des ressources en eau potable.

1. La diminution de l'eau douce disponible va entrainer une baisse du débit des rivières sans diminuer sensiblement le flux de pollution dû à l'activité humaine, la concentration résultante sera donc plus élevée.

Inversement, pour certains paramètres, tels que hydrocarbure ou métaux lourds entrainés par le lessivage des chaussées ou les excédents des traitements agricoles (pesticides, engrais) leur apport au réseau hydrique diminuera en l'absence de précipitation. Ce phénomène qui minimise l'effet polluant des baisses de débit souligne bien la complexité de l'évaluation d'un événement comme la sécheresse qui a des retentissements sur de multiples facteurs dont certains à effets contradictoires.

2. Le déficit de l'alimentation naturelle des nappes aquifères va entraîner une baisse de la pression piézométrique et une attraction accrue des eaux superficielles, dans ces circonstances, exceptionnellement polluées.

\section{Qualitative consequences of the drought on supply of drinking water}

The impact of the drought on the quality of water supplies is particularly hard felt in relation to superficial flowing waters.

On the one hand the direct impact can be seen in the concentration of pollution and, on the other hand, the indirect impact through the consequences of intense sunshine, favouring a biological explosion, stimulated at the same time by the concentration of nutriments. This bio-acceleration leads to degradation of resources, notably due to the abundance of special dissolved organic matter giving rise to unpleasant odours and tastes. These faults result from or generate operating difficulties for drinking water systems.

The anomalies observed in consumption in 1976 in the Paris region illustrate these difficulties very well. 
3. La sécheresse enfin, correspond à un ensoleillement intense et les micro-organismes, essentiellement les algues, gorgées d'éléments nutritifs (azote et phosphore concentrés par la pénurie d'eau), baignées de lumière solaire, vont croître d'une façon explosive diffusant dans le milieu une large gamme de composés organiques plus ou moins suspects de toxicité et de goût fâcheux.

Ce sont ces trois aspects des conséquences de la sécheresse que nous allons traiter dans le présent document à la lumière des nombreux résultats analytiques collectés en 1976 dans le bassin Seine Normandie.

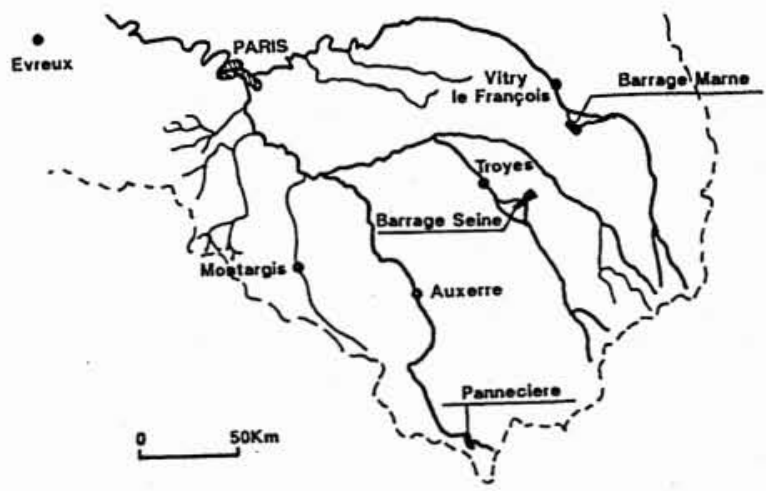

1. Carte des barrages réservoir.

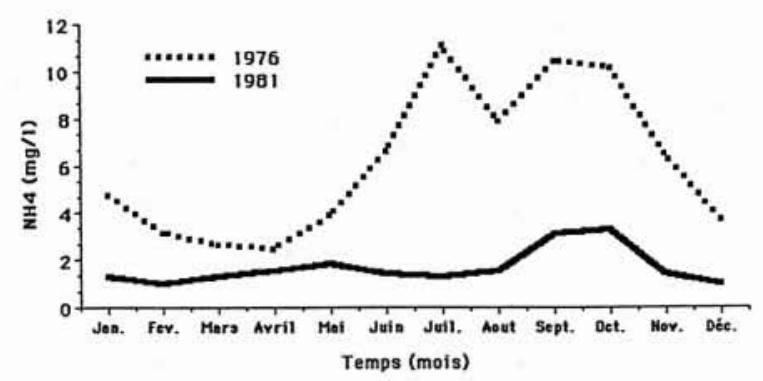

2. $\mathrm{NH}_{4}$ dans les eaux de la Seine à Poissy.

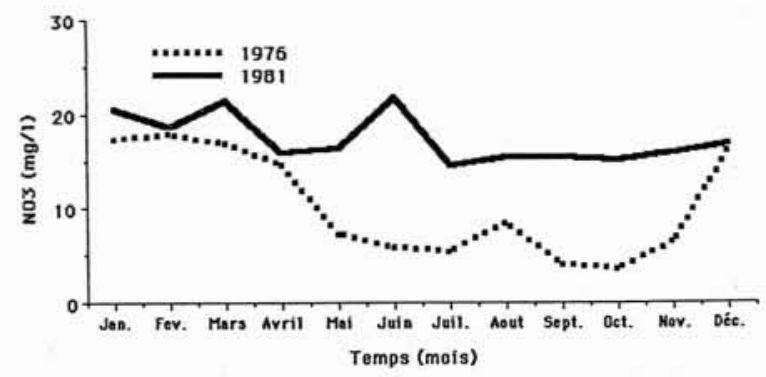

3. $\mathrm{NO}_{3}$ dans les eaux de la Seine à Poissy.

\section{Concentration du flux de pollution dans la Seine en 1976}

Malgré la mise en place d'énormes moyens palliatifs aux étiages sévères (fig. 1), le débit de la Seine tombé à quelques $\mathrm{m}^{3} / \mathrm{s}$ au cours de l'été 76 était du même ordre de grandeur que les principaux débits de pollution, Achères notamment $\left(16 \mathrm{~m}^{3} / \mathrm{s}\right)$. Les taux de phosphore et d'azote atteignent alors des valeurs record (Inventaire 1976 de la pollution) (réseau 61 campagne 83).

Ex.: Ammonium et orthophosphates atteignent à Conflans-Sainte-Honorine en juin 76 des valeurs triples de celles observées à la même époque en 83 .

Le problème de l'azote est particulièrement complexe en raison de l'oxydation bactérienne dont est l'objet le « rejet azote » dans le milieu naturel.

En période estivale sèche, il se fait un verrouillage du système : la chaleur et la concentration en substrat azoté exceptionnellement élevées accélèrent l'oxydation bactérienne et par conséquent la demande en oxygène due à cette réaction $(3,4 \mathrm{mg}$ d'oxygène pour transformer $1 \mathrm{mg}$ d'azote ammoniacale en azote nitrique) (MARTIN G., 1979). Simultanément la biodégradation des matières organiques à tendance à s'emballer et accroître la demande en oxygène alors que la baisse de débit et l'élévation de température appauvrissent l'eau en oxygène suivant des mécanismes physiques. Les bioxydations sont donc bloquées et l'azote s'accumule sous forme ammoniacale qui met la faune piscicole d'autant plus en danger que le $\mathrm{pH}$ et la température sont élevés (EIFAC, 1978).

Pour la ressource en eau on observe donc une spectaculaire surconcentration en ammoniaque (fig. 2), tandis que le taux de nitrate diminue (fig. 3). La filière de potabilisation doit alors faire face à un flux important d'ammoniaque à nitrifier. La température est alors favorable et l'air peut être fourni industriellement en abondance (RICHARD Y. et coll., 1978).

De même que l'azote et le phosphore, la charge carbonée des rivières augmente en concentration par un mécanisme analogue. Ceci aggrave la demande en oxygène.

Plus généralement on assiste donc à une surconcentration exceptionnelle des résidus de la vie " animale".

Par contre, la surconcentration en éléments résultant de l'activité industrielle n'est pas toujours aussi nette.

- en raison des faibles valeurs habituellement observées, la concentration peut croître relativement tout en restant inférieure aux valeurs mesurables et rester le plus souvent, en dessous du seuil analytique (fig. 4);

- par ailleurs, la baisse de l'activité industrielle au cours des mois d'été (juillet-août) se traduit par une baisse des rejets qui minimise, au moins, sur les grandes rivières, l'effet de l'appauvrissement hydrique du milieu récepteur.

La dégradation qualitative de la Seine, dépistée en 1976 , a donc essentiellement concerné les matières organiques, l'azote et le phosphore. Les traceurs spécifiques de pollutions d'origine industrielle ou agricole n'ont pas atteint les cotes alarmantes par suite d'événements annexes : ralentissement estival de l'activité, absence de ruissellement, inadaptation de l'outil analytique. 


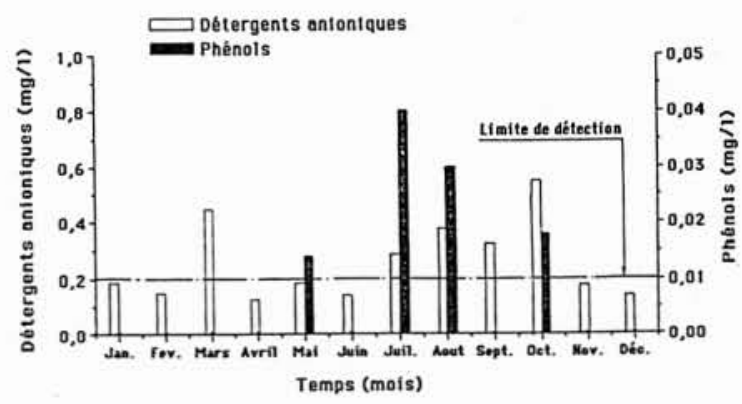

4. Pollution résultant de l'activité humaine. Seine à Poissy en 1976.

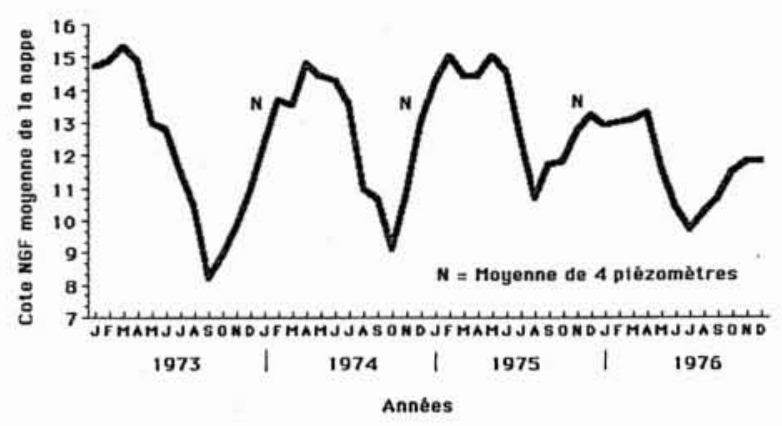

5. Niveaux piézométriques moyens de la nappe de Croissy de 1973 à 1976

Tableau 2. Teneur en ammonium. $1976(\mathrm{~g} / \mathrm{l})$

\begin{tabular}{|c|c|c|c|}
\hline & $\underbrace{}_{\substack{\text { Janviler } \\
\text { Avri1 }}}$ & Juillet & octobre \\
\hline Seine à croissy & 3,2 & 11 & 6,2 \\
\hline $\begin{array}{l}\text { Forage partielle- } \\
\text { ment influencé par } \\
\text { la réalimentation }\end{array}$ & 0,15 & 0,95 & 1,6 \\
\hline $\begin{array}{l}\text { Forage très in- } \\
\text { fluence par la } \\
\text { rêalimentation }\end{array}$ & 0,0 & 0,2 & 0,2 \\
\hline
\end{tabular}

\section{Impact de la sécheresse sur la qualité des eaux de} nappe aquifère

Au niveau des nappes aquifères comme à celui des rivières, la sécheresse se traduit par un défaut de l'alimentation. $\mathrm{Si}$ la nappe est sollicitée par puits ou par forage pour l'alimentation en eau potable, cette situation d'alimentation restreinte s'aggrave d'une consommation et donc d'une exploitation accrue du gîte aquifère en période sèche. $\mathrm{La}$ conjugaison de ces deux tendances va se traduire par une baisse du niveau piézométrique de la nappe et donc, une attraction accrue des eaux avoisinantes. Ces eaux plus polluées qu'à l'habitude par suite des phénomènes exposés en 1 vont pénétrer dans les nappes et en compromettre la qualité.

Un excellent palliatif à cette pollution est, quand le site le permet, la réalimentation artificielle de la nappe aquifère considérée. Cette technique permet de remonter le niveau piézométrique (fig. 5) pour limiter les infiltrations tout en maîtrisant la qualité des eaux introduites (tabl.2).

Ainsi en 1976 la quantité d'eau introduite dans la nappe de Croissy en réalimentation artificielle de juin à octobre a représenté le double de la quantité infiltrée l'année précédente et atteint 13 millions de $\mathrm{m}^{3}$ (cf. tabl. 3).

\section{Pollution résultant d'une accélération explosive de la croissance des biocénoses aquatiques}

Avec l'usage immodéré des engrais naturels ou chimiques et le transfert au système hydrique, les larges excédents d'azote et de phosphore, les notions d'eutrophisation, de production primaire, de chaînes alimentaires sont entrées dans le langage courant. Elles y figurent avec un sens plus ou moins authentique, symbole, en tout cas, des conséquences fatales de l'incontrolable application des "plus " scientifiques.

La sécheresse et son cortège d'ensoleillement intense et de surconcentration en éléments nutritifs va jouer le rôle d'accélérateur vis-à-vis des micro-organismes aquatiques. Cette accélération va commencer par la base de la chaîne alimentaire, la production primaire va s'intensifier. Cette intensification va se traduire par une prolifération du phyto-plancton (petites algues d'eau libre). Cette surcrois-

Tableau 3. Réalimentation (Croissy-Le Pecq). Total des $\mathrm{m}^{3}$ injectés par mois

\begin{tabular}{|c|c|c|c|c|c|c|c|c|c|c|c|}
\hline Janvier & Pévrier & Mars & Avril & $\mathrm{mai}$ & Juin & Juillet & Août & Septembre & Octobre & Novembre & Décembre \\
\hline 19752.626 .300 & 1.620 .300 & 1.809 .100 & 2.595 .000 & 2.862 .200 & 2.396 .100 & 104.600 & 1.165 .700 & 1.342 .900 & 1.733 .400 & 3.188 .800 & 2.513 .300 \\
\hline 19762.578 .800 & 1.529 .400 & 2.295 .500 & 2.420 .400 & 2.713 .000 & 2.956 .200 & 2.998 .300 & 2.228 .300 & 2.239 .200 & 2.634 .600 & 2.265 .800 & 2.583 .000 \\
\hline $8-1,8$ & $-5,6$ & $+26,9$ & $-6,7$ & $-5,2$ & $+23,4$ & $+2.766,00$ & $+91,2$ & $+66,7$ & $+52,0$ & $-\quad 28,9$ & $+2,8$ \\
\hline
\end{tabular}

Période de réalimentation de la nappe. 


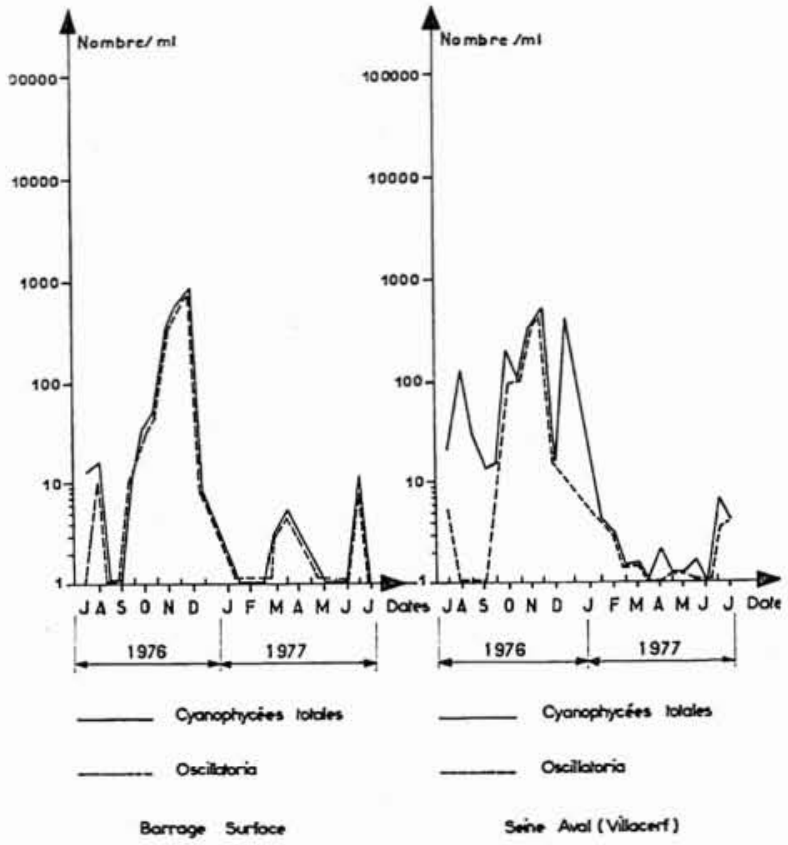

sance sera d'autant plus importante que les eaux seront dormantes. Or, l'absence de courant dans les rivières est une conséquence de la sécheresse, c'est également une conséquence des grands réservoirs, aménagés pour pallier aux effets quantitatifs de la sécheresse.

La multiplication du nombre d'individus algaux va s'accompagner d'une diffusion intense dans le milieu des molécules organiques qu'elles synthétisent, et d'un appauvrissement du nombre des espèces présentes.

La richesse en matière organique va favoriser certains groupes d'organismes tolérants à cet égard, dont les cyanophycées ou algues bleues. Certaines espèces de ce groupe représentent une véritable peste aquatique en raison des métabolites à goût de terre et de moisi qu'elles génèrent, leur résistance aux algicides et leur faible prédation.

Au cours de l'été et de l'automne 1976 il a été détecté jusqu'à 1000 oscillaires au $\mathrm{cm}^{3}$ dans les eaux du réservoir "Seine» (fig. 6) (Rizet M., Mouchet J.). Simultanément, la plupart des filières de potabilisation des eaux de Seine en région parisienne devait faire face à l'élimination de goût de moisi, particulièrement désagréable aux consommateurs. Les molécules organiques responsables de ces goûts ont été identifiées (Montiel A., 1979) comme étant de la géosmine et du diméthyl isobornéol (fig. 7).

Ces molécules, bien absorbées sur charbon actif en grains, demandaient pour une efficacité suffisante vis-à-vis des goûts, des taux de traitement en charbon actif en poudre qui dépassaient parfois la capacité de traitement des filières en place.

Une conséquence de l'été 1976 a été l'accélération de la mise en place des réacteurs à charbon actif en grains dans
6. Cyanophycées dans le barrage et la Seine aval.

7. Géosmine et Méthyl isobornéol.
Géosmine<smiles>CC1CCC[C@@]2(C)CCCCC12O</smiles>

(M.I.B.)

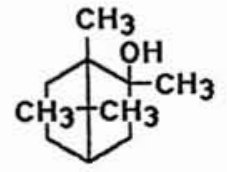

Méthyl isobornéol

les filières de traitement des eaux de Seine. L'optimisation de la gestion des barrages réservoirs pour maîtriser l'accumulation des sédiments et les échanges biochimiques avec la masse d'eau stockée n'est pas encore achevée.

\section{Conclusion}

Il s'est avéré à la lumière de la sécheresse subie en 1976, que les mesures prises pour pallier quantitativement à un tel événement ont eu de fâcheuses conséquences qualitatives. Des mesures ont été prises au niveau des filières de potabilisation elle-même pour éliminer les molécules responsables de goût désagréable (couplage ozone/charbon actif en grains). La maîtrise des phénomènes biologiques générateurs de ces molécules ne paraît pas encore parfaitement réalisée.

En ce qui concerne le flux polluant déversé en Seine, de nombreuses mesures orchestrées par l'Agence pour l'eau, ont permis au cours des années passées de le réduire en généralisant l'utilisation des traitements d'épuration, et en améliorant leur rendement épuratoire. Des progrès restent à faire dans ce domaine pour limiter les flux d'azote et de phosphore générés par la grande majorité des stations en place.

La réalimentation artificielle de la nappe aquifère s'est révélée d'une précieuse efficacité en permettant le stockage d'eau de bonne qualité à l'abri des vicissitudes climatiques (chaleur, température), et en limitant les intrusions d'eaux superficielles polluées par une bonne tenue des niveaux piézométriques. 


\section{Références bibliographiques}

European Inland Fisheries Advisory Commission. - Technical Paper, $\mathrm{n}^{\circ} 11,1978$.

Inventaire du degré de pollution des eaux superficielles (rivières et canaux) - Campagne 1976 - Bassin Seine-Normandie. Ministère de la Culture et de l'Environnement, vol. III (387 pages).

MARTIN G. et coll. - Le problème de l'azote dans les eaux. Technique et documentation, 1979 (279 pages).

Montiel A. - Détermination des principaux métabolites d'algue et d'actinomycète responsables des goûts de terre et de moisi dans les eaux. Informations chimiques, 1979 (pp. 2528).

Réseau 61 - Campagne 1983. - Surveillance de la qualité des eaux à l'amont des prises d'eau de la Région parisienne. Agence financière de Bassin Seine-Normandie.

Richard Y., Brener L., Martin G., Leblanc Ch. - Study of the nitrification of surface water. Prog. Wat. Tech., 1978, vol. $10, \mathrm{n}^{\circ} 5 / 6$, pp. 17-32, Pergamon Press.

Rizet M. et Mouchet Y. - Influence of discharges from storage reservoirs on the tastes and odours appearing in the Seine and Marne rivers. Wat sci. Tech., vol. 14, Capetown, pp. $43-58,1982$. 